\title{
Deployment Analysis of cooperative OFDM base stations
}

\author{
Gaoning He, Hamidou Tembine and Mérouane Debbah
}

\begin{abstract}
We study the resource allocation problem in Orthogonal Frequency Division Multiplexing (OFDM) systems as a cooperative game. The goal is to maximize the overall system rate considering fairness metrics among users. We propose Nash bargaining solution (NBS) as a tool that achieves point(s) on the Pareto frontier of the game theoretical rate region under asymmetric conditions. Moreover, we provide a practical stochastic algorithm that can converge to one of the fairness points on the Pareto boundary. The numerical results show that the NBS not only maintains fair resource allocation for all users, but also provides a desirable spectral efficiency for the OFDM system.
\end{abstract}

\section{INTRODUCTION}

There has been a recent interest in small cells [1] where people can access Internet over many "mini" base stations (hot-spots). Typically in such a wireless network, multiple "mini" base stations are settled to cover a small area (e.g. airports, restaurants, military bases, hotels, hospitals, libraries, supermarkets, etc.). In this paper, we assume that these base stations are communicating simultaneously to several receivers using OFDM (Orthogonal Frequency Division Multiplexing) over a number of dedicated sub-channels. It is well known that an $N$-carrier OFDM system [2] using a cyclic prefix or zero-padding [3], [4] to prevent interblock interference is equivalent in the frequency domain to $N$ flat fading parallel transmission channels. This enables a power allocation on a carrier basis. In such an interferencelimited multiuser communication environment, power control becomes a central issue in the system design, since each user's performance depends not only on its own transmit power, but also on the transmit power of all the other users.

In general, there are two ways to study the power allocation problem depending on the system settings, i.e. centralized approaches and decentralized approaches.

In centralized approaches, the problem (thanks to a central scheduler) is considered as a global optimization problem in order to maximize the overall achievable sum-rate (corresponding to the Shannon capacity [5] when single user detecting is applied). From a single user point of view, given other users' states and power allocation strategies, it is a classical convex optimization problem [6], whose solution is given by "water-filling" [7], [8], [9]. The multi-user version of this problem is a non-convex optimization problem where there may exist multiple local optimal points. Unfortunately

G. He is with Télécom ParisTech, 75013 Paris, France. gaoning.he@gmail.com

H. Tembine is with University of Avignon, 84911 Avignon, France. hamidou.tembineduniv-avignon. fr

M. Debbah is with SUPELEC, 91193 Gif-sur-Yvette, France. merouane.debbahesupelec.fr it can be shown that this kind of problem is NP-hard [10] and often difficult to solve efficiently for an exact global optimum. Some efforts have been made in this direction to achieve a reasonable trade-off between the global performance and the computational complexity, as in [11]. As a matter of fact, the major problem for the centralized approach is that the system requires a central computing resource with complete and probably perfect knowledge of the channel state information (CSI), involving feedback and overhead communication whose load scales linearly with the number of transmitters and receivers in the network. Due to fading and high mobility, it becomes computationally expensive for many current wireless systems to track the complete and perfect CSI. Some other efforts have been made to find a satisfying trade-off between the global performance and the feedback load while maintaining a reasonable computational complexity, e.g. communication with partial feedback [12]. However, the performance of centralized approaches is often limited by the condition of communication environment and the scaling laws.

Very different from centralized approaches, decentralized approaches aim to share the global computing and operating tasks to every entity in the network without setting an organized center or authority. In this respect, game theory [13] has been considered as a powerful mathematical tool to analyze "optimal solution" in the competitive and cooperative environments.

When the hot-spots belong to different network providers, non-cooperative games appear to be a natural setting for modeling the competition between providers in such networks. The central question here is whether a Nash equilibrium (NE) exists (or under which conditions NE exists), and if so, whether the system operates efficiently at the NE. One of the earliest contributions in this direction is the work of $\mathrm{Yu}$ et. al [14]. With a simplified two-user interference channel model, they show that the existence and uniqueness of Nash equilibrium is satisfied only under certain conditions (depending on the channel crosstalk matrix). The iterative water-filling (IWF) algorithm is proposed to find an approximate solution by splitting the original problem into several convex sub-problems, then iterating over them. IWF is amenable to practical implementation without the need for a centralized control. As a multiuser extension, [16] shows that for arbitrary symmetric interference environments and for certain asymmetric channel conditions, IWF can converge to a competitive NE. A more general extension on the channel gain matrix has been made in [17]. It shows that a unique NE almost surely exists, if the channel gain matrix is assumed to be "random". Fortunately this assumption 
is usually valid for wireless channels, due to independent fadings. However, sustained by numerical results, [17] shows that the inefficiency of non-cooperative game approaches can be significant comparing to the centralized approaches, especially when the number of transmitters increases. Moreover, the performance unbalance between users can be a serious problem, since there is absolutely no control on the fairness issue. Similar results can be found in [11], where the authors show that the performance of IWF can be improved by up to $380 \%$ in their centralized approach. Obviously the efficiency of "competitive optimality" is not so inspiring from an engineering point of view.

However, in many practical applications, the hot-spots belong to the same network provider. In this situation, providers may prefer a full cooperation of the hot-spots with limited signalings (due to the lack of a backbone infrastructure) in order to obtain cooperative gains and further improve the performance from NE. Therefore, it is natural to extend the framework of non-cooperative games to cooperative games. In this paper, we take into consideration the concept of the NBS, as it provides a fair operation point for cooperative communication systems. The NBS is a standard tool in cooperative game theory, and is applied widely in network resource allocation. For example, in [18], a criterion based on NBS is applied to orthogonal frequency division multipleaccess (OFDMA) networks. This method generalizes the proportional fairness and increases the efficiency of the system. In [19], the ideas of max-min fairness, proportional fairness and NBS have been applied to the Gaussian multiple access channel (MAC) and the Gaussian broadcast channel (BC). Algorithms have been designed to locate the fair point in the capacity region. In [20], cooperative game is used to provide preferred points on the boundary of the achievable rate region for a simple $2 \times 2$ interference channel. From their simulation results, the cooperative solution NBS is shown to significantly outperform the competitive NE.

The aim of this study is to design cooperative algorithms to maximize the overall system rate considering fairness between players, under the constraints of players' minimal rate requirements and maximal transmit powers.

The paper is organized as follows. In section II, we describe the system model and the resource allocation problem. In section III, we characterize asymmetric NBS with its efficiency. Finally, numerical results are provided in section IV followed by a conclusion in section V.

\section{System Model AND DESCRIPTION}

In this section, we describe the system model considering $N$ independent parallel Gaussian channels with $M$ transmitters simultaneously communicating to $N$ receivers. We define the $M$ transmitter set as $\mathcal{M}:=\{1,2, \ldots, M\}$ and the $N$ sub-channel set as $\mathcal{N}:=\{1,2, \ldots, N\}$. We assume that each receiver is connected to several hot-spots (from which it decodes data) on a pre-assigned carrier. It can download information from several hot-spots simultaneously. Moreover, all receivers detect signals in different bands (a carrier),

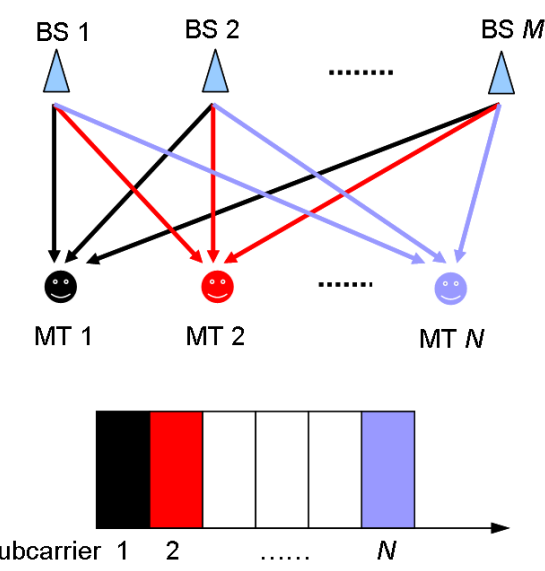

Fig. 1. The multi-user multi-carrier channel model

as shown in Fig. 1. For wireless communication systems, typically we assume that the sub-channels have slow-fading so that the channel fading coefficients are constant during the period of each OFDM frame. Within a given OFDM frame, let $\mathbf{G} \in \mathbb{R}_{+}^{M \times N}$ be the channel gain matrix where the $(m, n)$ entry of $\mathbf{G}$ is denoted as $g_{m, n}$, the magnitude-squared of the fading channel gain for the downlink channel from the $m^{\text {th }}$ base station (BS) to the $n^{\text {th }}$ MT. G can be modeled as a random matrix chosen from a jointly Rayleigh distribution. We also assume that each MT decodes the information from each BS using single user detector, i.e. when MT $n$ detects BS $m$ 's signal, it treats the signals from other base stations as noise. Therefore, the signal to interference plus noise ratio (SINR) from BS $m$ to MT $n$ is defined as

$$
\gamma_{m, n}=\frac{g_{m, n} p_{m, n}}{\sigma^{2}+\sum_{j \neq m} g_{j, n} p_{j, n}}, \quad \forall m \forall n
$$

where $\sigma^{2}$ models the white Gaussian noise variance on each subcarrier $n, p_{m, n} \geq 0$ represents the power transmitted by the $m^{\text {th }}$ BS on the $n^{\text {th }}$ subcarrier, and the corresponding maximum achievable rate (known as capacity) with simple decoder at the receiver side is given by [7]

$$
R_{m, n}=\log \left(1+\gamma_{m, n}\right), \quad \forall m \forall n
$$

Hence, the sum-rate for each BS $m$ is

$$
R_{m}=\sum_{n=1}^{N} R_{m, n}=\sum_{n=1}^{N} \log \left(1+\gamma_{m, n}\right), \quad \forall m
$$

which represents the intuitive "utility" in a non-cooperative game setting [17]. The total transmit power of each BS is restricted by a power constraint

$$
\sum_{n=1}^{N} p_{m, n} \leq \bar{P}_{m}, \quad \forall m
$$

for $\bar{P}_{m}>0, \forall m$. Note that the non-cooperative setting of (3) has been studied in [17]. However, here we focus on the cooperative setting for which we introduce the Nash Bargaining concept. 


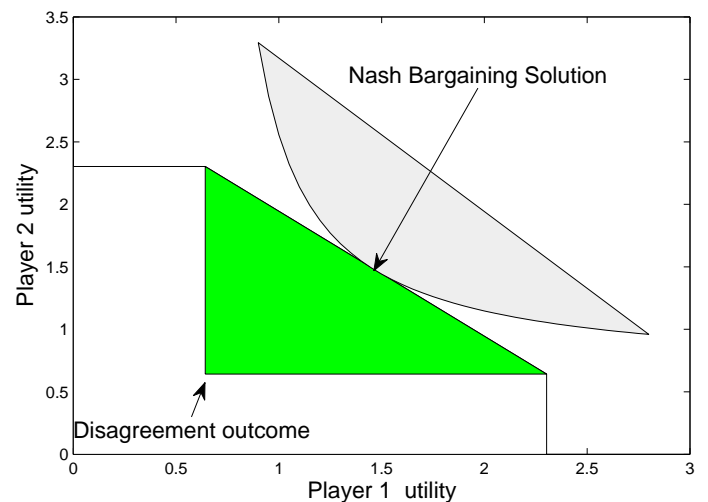

Fig. 2. Illustration of Nash bargaining solution

\section{ASYMMETRIC NASH BARGAINING}

\section{A. Nash Bargaining definition}

The Nash bargaining is a natural framework that allows us to define and design a fair assignment of rate between players which will play the role of bargainers. It is characterized by a set of axioms that are appealing in defining fairness or by a maximization of log-concave function on the set of feasible sum-rates. We are interested in the NBS since it can be seen as a natural extension of the proportional fairness criterion which is probably the most popular fairness. Players are faced with the problem to negotiate for a fair point in the convex set of feasible rates. If no agreement can be achieved by the players, the disagreement utilities (sum-rate) is obtained (see Fig.2).

The standard Nash bargaining assumes that all players in the cooperative game have the same priority. The symmetry axiom ensures all players have the same priority. It assures that the players will achieve the same NBS, if they have the same feasible utility function set. The asymmetric Nash bargaining is identical to the NBS except that the symmetry axiom is not satisfied.

For a set of players $\mathcal{N}$, a Nash bargaining game is defined by a pair $\left(R_{m}^{\min }, \mathcal{F}\right)$, where $\mathcal{F}$ is a compact and convex set which defines the feasible set of utilities of all the agents, and $\left(R_{m, n}^{\min }\right)_{m, n} \in \mathcal{F}$ is known as the disagreement point which defines the amount of utility each player will get if the bargaining process fails. Whenever the disagreement situation can be decided by a non-cooperative game, it is reasonable to assume that the disagreement rate solution is given by a NE of the relevant non-cooperative game. When the utility for player $m$ is given by the rate $R_{m}(p)$ in (3), and $p^{*}$ is the power allocation matrix at the NE of the noncooperative game (which can be obtained by IWF [16]), then $R_{m}^{\min }$ can be taken as

$$
R_{m}^{\min }=R_{m}\left(p^{*}\right)=\sum_{n=1}^{N} \log \left(1+\gamma_{m, n}\left(p_{n}^{*}\right)\right), \forall m
$$

the worse Nash equilibrium payoff in the non-cooperative case, where vector $p_{n}^{*}$ represents the $n^{t h}$ column of $p^{*}$. The asymmetric bargaining solution $\left(R_{m}\right)_{m} \in \mathcal{F}$ satisfies the following conditions:
- Pareto optimality ${ }^{1}$.

- individual rationality ${ }^{2}$.

- Invariance to positive affine transformations.

- If the domain $\mathcal{F}$ is reduced to a subset of the domain that contains the bargaining solution, then the bargaining solution remains the same.

$$
\max _{\substack{R, R_{m, n} \geq R_{m, n}}} \prod_{m=1}^{M}\left(R_{m}-R_{m}^{\min }\right)^{\omega_{m}}
$$

subject to

$$
\mathcal{F}=\left\{\begin{array}{c}
\forall n \in \mathcal{N} \\
\forall \emptyset \neq \mathcal{J} \subseteq\{1,2, \ldots, N\}: \mathcal{J} \in 2^{\mathcal{N}} \backslash\{\} \\
R_{m, n} \geq 0 \\
\sum_{m \in \mathcal{J}} R_{m, n} \leq \log \left(1+\frac{\sum_{m \in \mathcal{J}} p_{m, n} g_{m, n}}{\sigma^{2}}\right)
\end{array}\right.
$$

where $\omega_{m} \geq 0$ is the weight of "bargaining power" (which is related to the relative abilities of players in a situation to exert influence over each other) for player $m$ in the interaction.

Note that the outcome (rate set) of (5) may not be a feasible "instantaneous" solution for the transmit power (4). However, it is known that any point in the rate region can be achieved by a simple time or frequency sharing strategy [9]. We now focus on the problem of rate Nash bargaining.

\section{B. Transformation to concave optimization problem}

In order to find the rate NBS, we consider the non-concave maximization problem (5), which can be actually transferred to a convex optimization:

$$
\max _{\substack{R \in \mathcal{F} \\ R_{m, n} \geq R_{m, n}^{\min }}} \sum_{m \in \mathcal{M}} \omega_{m} \log \left(R_{m}-R_{m}^{\min }\right)
$$

where the objective function is concave in $R$ and the constraint space $\mathcal{F}$ is non-empty, convex and compact.

Hence, for a fixed value of disagreement point (for example, the disagreement is equal to the worse Nash equilibrium payoff), the asymmetric Nash bargaining solutions, i.e. $R^{N B S}=\left\{R_{1}^{N B S}, \ldots, R_{M}^{N B S}\right\}$, can be found by solving the following convex optimization problem:

$$
R^{N B S}=\arg \max _{\substack{R \in \mathcal{F} \\ R_{m}>R_{m}^{\min }}} \sum_{m \in \mathcal{M}} \omega_{m} \log \left(R_{m}-R_{m}^{\min }\right)
$$

Note that the uniqueness is only on the total rate in all the subcarrier of each player. Rate per subcarrier needs not be unique. Therefore, for each player, the bargaining solutions over all the subcarriers will lead to the same utility. But players may have different utilities. The reason of nonuniqueness of the bargaining solution is that the function $R_{m}(\cdot)$ is not injective.

We regularize the logarithm of the product (sometimes referred to Nash product) by substituting the logarithmic

\footnotetext{
${ }^{1}$ An allocation of payoffs is Pareto optimal or Pareto efficient if there is no other feasible allocation that makes every player at least as well off and at least one player strictly better off.

${ }^{2}$ Each player has at least the disagreement payoff.
} 
function with the following function, defined for all $r \in \mathbb{R}$ by

$$
\phi_{\epsilon}(r)=\left\{\begin{array}{cc}
\log (\epsilon)+\frac{r-\epsilon}{\epsilon} & \text { if } r \leq \epsilon \\
\log (r) & \text { if } r>\epsilon
\end{array}\right.
$$

This regularization replaces the logarithm function by its Taylor first order development for small rates. We then have a regular function to maximize in all the feasible sum-rate set. The following Lemma shows that this regularization gives the same bargaining solutions.

Lemma III.1. For $\epsilon>0$ sufficiently small, equation (6) is equivalent to the following expression :

$$
\max _{R \in \mathcal{F}} \sum_{m \in \mathcal{M}} \omega_{m} \phi_{\epsilon}\left(R_{m}-R_{m}^{\min }\right)
$$

Proof: See in appendix $A$.

Note that the expression (8) in Lemma III.1 maximizes over the entire set $\mathcal{F}$ of feasible and individual rational rate profile. This result is crucial to gradient-based algorithms to not have to account for scenarios where a BS currently achieves a utility lower than the disagreement point, which may happen before the algorithm converges. We will be able to guarantee to each BS at least the worse Nash equilibrium rate at any step of the adaptive algorithm.

\section{Karush-Kuhn-Tucker optimality conditions}

It is easy to verify that the sum-log function is concave and the corresponding constraint set is convex. So, the Karush-Kuhn-Tucker (KKT) condition of the optimization is sufficient and necessary for the optimality [6]. To derive the KKT conditions, form the Lagrangian function $\mathcal{L}$ as:

$$
\begin{aligned}
\mathcal{L}(R, \lambda)= & \sum_{m \in \mathcal{M}} \omega_{m} \log \left(\sum_{n \in \mathcal{N}} R_{m, n}-R_{m}^{\min }\right) \\
& -\sum_{\mathcal{J} \subseteq \mathcal{M}} \sum_{n \in \mathcal{N}} \lambda_{n}^{\mathcal{J}}\left(\sum_{m \in \Omega_{\mathcal{J}}} R_{m, n}-\bar{R}_{n}^{\mathcal{J}}\right)
\end{aligned}
$$

where $\bar{R}_{n}^{\mathcal{J}}$ represents the maximum sum-rate on subcarrier $n$ for the sub-group of players $\mathcal{J}, \lambda_{n}^{\mathcal{J}} \geq 0$ is the dual variable for the associated sum-rate constraint, and the KKT conditions are

$$
\begin{aligned}
\frac{\omega_{m}}{\sum_{n \in \mathcal{N}} R_{m, n}^{\star}-R_{m}^{\min }}-\beta_{n} & =0, \forall m \forall n \\
\lambda_{n}^{\mathcal{J}}\left(\sum_{m \in \mathcal{J}} R_{m, n}^{\star}-\bar{R}_{n}^{\mathcal{J}}\right) & =0, \forall n \forall \mathcal{J}
\end{aligned}
$$

where the optimal rate set $R^{\star}$ represents the sum-rate bargaining solution $R^{N B S}$ in (7), and we define $\beta_{n}:=$ $\sum_{\mathcal{J}, m \in \mathcal{J}, \mathcal{J} \subseteq \mathcal{M}} \lambda_{n}^{\mathcal{J}}$. Note that two observations can be made based on (9):

- First, all the $\beta_{n}$ have the same value, and we can define $\beta:=\beta_{1}=\beta_{2}=\ldots=\beta_{N}$.

- Second, it is easy to see that $\beta>0$, since $\omega_{m}>0, \forall m$.
Proposition III.1. The Lagrangian multiplier $\lambda_{n}^{\mathcal{M}}$ is positive for all $n$, and at the optimality point, the largest rate constraint must be satisfied with equality, i.e.

$$
\sum_{m \in \mathcal{M}} R_{m, n}^{\star}=\bar{R}_{n}^{\mathcal{M}}
$$

Proof: See in appendix $B$.

From KKT conditions we have the following linear equations

$$
\begin{aligned}
\sum_{m \in \mathcal{M}} R_{m, n}^{\star} & =\bar{R}_{n}^{\mathcal{M}}, \forall n \\
\sum_{n \in \mathcal{N}} R_{m, n}^{\star} & =R_{m}^{\min }+\omega_{m} \beta^{-1}, \forall m
\end{aligned}
$$

and the parameter $\beta$ can be calculated as

$$
\beta^{-1}=\sum_{n \in \mathcal{N}} \bar{R}_{n}^{\mathcal{M}}-\sum_{m \in \mathcal{M}} R_{m}^{\min }
$$

In general, equations (12), (13) have $M N+1$ variables (which are $\beta$ and all the $R_{m, n}$ ), but one only yields $M+N$ independent linear equations. This could simply imply that the optimization problem has multiple solutions. From Equation (13), we find that each player's sum-rate $\sum_{n \in \mathcal{N}} R_{m, n}^{\star}$ is unique at a bargaining solution. This confirms the uniqueness in sum-rate, but we may have multiple splitting rate solutions over the subcarriers.

\section{Pure Potential}

The concept of potential games were proposed by Monderer and Shapley in 1996. The idea is to find the potential function such that the incentive of all players are mapped into one global function, and the set of pure Nash equilibria can be found by simply locating the local optima of the potential function. In our problem, for a subgroup of players $\mathcal{J} \subseteq \mathcal{M}$, we define function

$$
v(\mathcal{J}):=\sum_{n \in \mathcal{N}} \log \left(1+\frac{\sum_{m \in \mathcal{J}} p_{m, n} g_{m, n}}{\sigma^{2}}\right)
$$

the maximum sum-rate that the coalition $\mathcal{J}$ receives. Then ,$v(\emptyset)=0$ and the following inequalities holds:

$$
v(\{m\})=\sum_{n \in \mathcal{N}} \log \left(1+\frac{p_{m, n} g_{m, n}}{\sigma^{2}}\right) \geq R_{m}
$$

For $\forall m \notin \mathcal{J}$, we define

$$
\begin{aligned}
\Delta v_{m, \mathcal{J}} & :=v(\mathcal{J} \cup\{m\})-v(\mathcal{J}) \\
& =\sum_{n \in \mathcal{N}} \log \left(1+\frac{p_{m, n} g_{m, n}+\sum_{m^{\prime} \in \mathcal{J}} p_{m^{\prime}, n} g_{m^{\prime}, n}}{\sigma^{2}}\right) \\
& -\sum_{n \in \mathcal{N}} \log \left(1+\frac{\sum_{m^{\prime} \in \mathcal{J}} p_{m^{\prime}, n} g_{m^{\prime}, n}}{\sigma^{2}}\right) \\
& =\sum_{n \in \mathcal{N}} \log \left(1+\frac{p_{m, n} g_{m, n}}{\sigma^{2}+\sum_{m^{\prime} \in \mathcal{J}} p_{m^{\prime}, n} g_{m^{\prime}, n}}\right) \\
& \geq R_{m} .
\end{aligned}
$$


Hence, for any $\mathcal{J} \subseteq \mathcal{J}^{\prime}$, we have

$$
\Delta v_{m, \mathcal{J}} \geq \Delta v_{m, \mathcal{J}^{\prime}}
$$

Note that when $\mathcal{J}=\mathcal{M} \backslash\{m\}$, the term $\Delta v_{m, \mathcal{J}}$ is exactly the sum-rate of mobile $m, R_{m}$. We say that $v$ is a pure potential. To see the link with static continuous-kernel noncooperative potential games, consider the difference

$$
\begin{aligned}
\Delta R_{m}:= & R_{m}\left(p_{m}, p_{-m}\right)-R_{m}\left(q_{m}, p_{-m}\right) \\
= & \sum_{n \in \mathcal{N}} \log \left(\sigma^{2}+\sum_{j \in \mathcal{M}} p_{j, n} g_{j, n}\right)- \\
& \sum_{n \in \mathcal{N}} \log \left(\sigma^{2}+q_{m, n} g_{m, n}+\sum_{j \in \mathcal{M} \backslash\{m\}} p_{j, n} g_{j, n}\right) \\
= & w\left(p_{m}, p_{-m}\right)-w\left(q_{m}, p_{-m}\right)
\end{aligned}
$$

where $w\left(p_{m}, p_{-m}\right)=v(\mathcal{M})$,

$w\left(q_{m}, p_{-m}\right)=\sum_{n \in \mathcal{N}} \log \left(1+\frac{q_{m, n} g_{m, n}+\sum_{j \in \mathcal{M} \backslash\{m\}} p_{j, n} g_{j, n}}{\sigma^{2}}\right)$

Next, we derive some relations between the pure potential and maximal feasible solutions (core). For $\mathcal{J}_{1}$ and $\mathcal{J}_{2}$ two subgroups of players, one has,

$$
\begin{aligned}
v\left(\mathcal{J}_{1}\right)+ & v\left(\mathcal{J}_{2}\right) \\
= & \sum_{n \in \mathcal{N}} \log \left[\left(1+\frac{\sum_{m \in \mathcal{J}_{1}} p_{m, n} g_{m, n}}{\sigma^{2}}\right)\right. \\
& \left.\left(1+\frac{\sum_{m \in \mathcal{J}_{2}} p_{m, n} g_{m, n}}{\sigma^{2}}\right)\right] \\
> & \sum_{n \in \mathcal{N}} \log \left(1+\frac{\sum_{m \in\left\{\mathcal{J}_{1} \cup \mathcal{J}_{2}\right\}} p_{m, n} g_{m, n}}{\sigma^{2}}\right) \\
= & v\left(\mathcal{J}_{1} \cup \mathcal{J}_{2}\right)
\end{aligned}
$$

This implies that $v$ is subadditive, i.e. $v\left(\mathcal{J}_{1} \cup \mathcal{J}_{2}\right)<v\left(\mathcal{J}_{1}\right)+$ $v\left(\mathcal{J}_{2}\right)$.

Using theses properties, the set of feasible utilities with maximal value for $\mathcal{M}$, i.e $\sum_{m \in \mathcal{M}} R_{m}=v(\mathcal{M})$, satisfies $\forall \mathcal{J} \subset \mathcal{M}, \sum_{m \in \mathcal{J}} R_{m} \leq v(\mathcal{J})$. This set is sometimes referred to the core. We transform the inequalities that define the core to a linear program (LP) problem:

$$
(\mathrm{LP}):\left\{\begin{array}{cc}
\max & \sum_{m} R_{m} \\
\text { s.t. } & R \in \mathcal{F}
\end{array}\right.
$$

Then, the non-emptiness of the core is exactly equivalent to the optimum value of the primal problem (21) (with LP feature) is equal to $v(\mathcal{M})$. The dual problem, through Lagrangian multipliers, can be written as:

$$
\begin{aligned}
& \mathcal{L}(R, \lambda, \mu) \\
& \quad=\sum_{m} R_{m}-\sum_{\mathcal{J}} \lambda_{\mathcal{J}}\left(\sum_{m \in \mathcal{J}} R_{m}-v(\mathcal{J})\right)+\sum_{m \in \mathcal{M}} \mu_{m} R_{m}
\end{aligned}
$$

The primal problem (LP) can be interpreted as

$$
\max _{R \in \mathbb{R}_{+}^{M}} \inf _{\substack{\lambda \geq 0, \mu \geq 0}} \mathcal{L}(R, \lambda, \mu)
$$

and the dual problem (DP) is obtained by inverting the "max" and "min":

$$
\inf _{\substack{\lambda \geq 0, \mu \geq 0}} \sup _{R \in \mathbb{R}_{+}^{M}} \mathcal{L}(R, \lambda, \mu)
$$

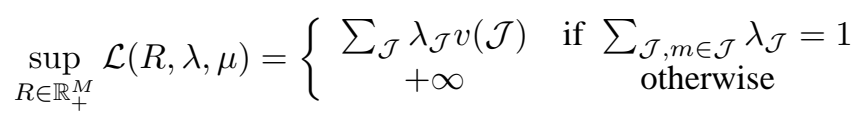

Thus,

$$
(\mathrm{DP}):\left\{\begin{array}{rr}
\min & \sum_{\mathcal{J}} \lambda_{\mathcal{J}} v(\mathcal{J}) \\
\text { s.t. } & \sum_{\mathcal{J}, m \in \mathcal{J}} \lambda_{\mathcal{J}}=1, \forall m \in \mathcal{J} \\
& \lambda_{\mathcal{J}} \geq 0, \forall \mathcal{J} \in 2^{\mathcal{M}}
\end{array}\right.
$$

\section{E. Stochastic Algorithm for Nash Bargaining}

In this part, we develop a recursive method of (8) to compute a Nash bargaining solution. Denote

$\mathcal{L}(R, \lambda)=\sum_{m} w_{m} \phi_{\epsilon}\left(R_{m}-R_{m}^{\mathrm{min}}\right)-\lambda_{n}^{\mathcal{M}}\left(\sum_{m \in \mathcal{M}} R_{m, n}-\bar{R}_{n}\right)$

A saddle point of the lagrangian $\mathcal{L}(\cdot, \cdot)$ is the pair $(R, \lambda)$ satisfying

$$
\mathcal{L}\left(R^{\prime}, \lambda\right) \leq \mathcal{L}(R, \lambda) \leq \mathcal{L}\left(R, \lambda^{\prime}\right), \quad \forall R^{\prime} \forall \lambda^{\prime}
$$

A necessary and sufficient condition for $R=\left(R_{m, n}\right)_{m, n}$ to be a constrained minimum is that there is a $\lambda$ with nonnegative components such that $(R, \lambda)$ is a saddle point of $\mathcal{L}(R, \lambda)$. Let us now consider a recursive algorithm for finding a saddle point. Let $R_{m, n}^{t}$ denote the $t^{t h}$ estimates and use the following form of the Robbins-Monro procedure. Let $\mathcal{C}$ denote the hypercube

$$
\prod_{m, n}\left[R_{m, n}^{\min }, \log \left(1+\frac{p_{m, n} g_{m, n}}{\sigma^{2}}\right)\right]
$$

The multipliers $\lambda$ are bounded because they are non-negative and their sum is upper-bounded (see equation (14)) by

$$
\frac{1}{\sum_{n \in \mathcal{N}} \log \left(1+\frac{\sum_{m \in \mathcal{M}} p_{m, n} g_{m, n}}{\sigma^{2}}\right)}
$$

From the KKT conditions, the constraint set can be reduced to

$$
\mathcal{C} \cap\left\{R, \sum_{m=1}^{M} R_{m, n} \leq \bar{R}_{n}, \forall n \in \mathcal{N}\right\}
$$

Denote

$$
q_{n}(R)=\bar{R}_{n}-\sum_{m=1}^{M} R_{m, n}
$$

The recursive algorithm is then given by

$$
\begin{aligned}
\lambda_{n}^{t+1} & =\max \left(0, \lambda_{n}^{t}+\epsilon^{t} q_{n}\left(R^{t}\right)+\epsilon^{t} \chi_{n}^{t}\right) \\
R_{n}^{t+1} & =\operatorname{proj}_{\mathcal{C}}\left[R_{n, t}+\epsilon^{t}\left(\mathcal{L}_{R}\left(R^{t}, \lambda^{t}\right)+\xi_{n}^{t}\right)\right]
\end{aligned}
$$

where the vector

$$
\begin{aligned}
R_{n}^{t} & =\left[R_{1, n}^{t}, \ldots, R_{M, n}^{t}\right] \\
\mathcal{L}_{R}\left(R^{t}, \lambda^{t}\right) & =\left[\frac{\partial}{\partial R_{1 n}^{t}} \mathcal{L}\left(\cdot, \lambda^{t}\right), \ldots, \frac{\partial}{\partial R_{M, n}^{t}} \mathcal{L}\left(\cdot, \lambda^{t}\right)\right]
\end{aligned}
$$


$\operatorname{proj}_{\mathcal{C}}$ denotes the projection operator over the hypercube $\mathcal{C}$, $\xi^{t}$ is the "noise" or "error" in the estimate of $\mathcal{L}(R, \lambda)$ and $\chi_{n}^{t}$ is the "noise" in the estimate of $q_{n}$. Using the regularized function $\phi_{\epsilon}$ in the Lagrangian, we obtain a regular concaveconvex function to maximize on the sum-rates and minimize on the $\lambda$. Using the methods in [15], the algorithm can be shown to converge to a saddle point under some conditions on the learning rates $\epsilon^{t}\left(\sum_{t} \epsilon^{t}=+\infty, \sum_{t}\left(\epsilon^{t}\right)^{2}<+\infty\right)$ with a good approximation of the noises.

\section{F. Strong Equilibria}

Since the definition of Nash equilibrium only requires the absence of any profitable unilateral deviations by any player. A Nash equilibrium is not guaranteed to be invulnerable to deviations by coalitions of players however. To study our problem, we will introduce the concept of Strong equilibria, proposed by Aumann in [21]. Note that the set of strong equilibria is a subset of Nash equilibria (by taking coalition of size one) and then any constrained strong equilibria is Pareto optimal (by taking coalition of full size).

We first describe the constrained strategic game $G$ defined as:

- The set of players is $\mathcal{M}$.

- The set of rates of player $m$ is $0 \leq R_{m, n}$ and $\sum_{n \in \mathcal{N}} R_{m, n}^{\prime} \leq \bar{R}_{m}$ such that in each subcarrier, any subgroup $\mathcal{J}$ of players are constrained to a common set

$$
\sum_{n \in \mathcal{N}} R_{m, n} \leq \log \left(1+\sum_{m \in \mathcal{J}} \frac{p_{m, n} g_{m, n}}{\sigma^{2}}\right)
$$

- If all the constraints are satisfied, each player $m$ receives $R_{m}$ otherwise they get zero.

A vector of rates is a $k$-strong equilibrium of $G$ if it is feasible and no coalition of size $k$ can improve the sum-rate of each of its members with the respect to the constraints in $\mathcal{F}$. A vector of sum-rates is a strong equilibrium if it is a $k$-strong equilibrium for any size $k=1, \ldots, M$. A strong equilibrium is then a splitting rate from which no coalition (of any size) can deviate and improve the sum-rate of every member of the coalition (group of the simultaneous moves), while possibly lowering the sum-rate of players outside the coalition group. This notion of strong equilibria is very attractive because it is resilient against coalitions of players. Most of the games do not admit any strong equilibrium.

Proposition III.2. The Nash bargaining solution for the sum-rate problem is a strong equilibria for $G$.

Proof: See in appendix $C$.

Moreover, the game $G$ has many strong equilibria, which will be described in Proposition III.3.

Proposition III.3. The set of strong equilibria of $G$ is $\left\{\left(R_{m, n}^{\prime}\right)_{m, n} \in \mathcal{F} \mid \sum_{n \in \mathcal{N}} \sum_{m \in \mathcal{M}} R_{m, n}^{\prime}=v(\mathcal{M}), R_{m, n}^{\prime} \geq R_{m, n}\right\}$

Proof: See in appendix $D$.
Note that we may not have strong equilibria in a strategic way in terms of transmission power. The following assertions are equivalent:

- $p^{*}=\left(p_{m}^{*}, p_{-m}^{*}\right)$ is a feasible transmit power set which gives a rate profile that satisfies (12) and (13).

- $p^{*}=\left(p_{m}^{*}, p_{-m}^{*}\right)$ is a strategic strong equilibrium.

\section{G. Efficiency and benefit of Bargaining}

We aim to measure the gap between the worse bargaining solution by varying the disagreement value $R^{\text {min }}$ and social optimum value (fully cooperative solution). Define the efficiency of sum-rate bargaining as the minimum over $\left(R^{\text {min }}, \mathcal{J}\right)$ of the ratio between the total sum-rate at the bargaining solution and the maximum value for the potential (15). It is easy to see that the ratio is less than one in a sum-rate bargaining solution. The efficiency of sumrate bargaining is greater than the sum-rate of competitive solutions (known as price of anarchy).

We introduce the benefit of bargaining $(\mathrm{BoB})$ to measure the gap (in percentage) between the worse bargaining solution and the worse competitive Nash equilibrium.

$$
B o B=\frac{\text { total-sum-rate }_{N B S}-\text { total-sum-rate }_{N E}}{\text { total-sum-rate }_{N E}}
$$

where total-sum-rate at NBS is defined as

$$
\sum_{m \in \mathcal{M}} R_{m}^{N B S}
$$

Since the worse competitive Nash equilibrium is an interior point of the capacity region, there is a gap between this point and the Pareto frontier. This means that the BoB in sum-rate is at least non-negative.

\section{NUMERICAL ILLUSTRATIONS}

In this section, we provide some numerical results to demonstrate the advantage of NBS regarding the average system performance. As a basis of comparison, the Nash equilibrium and the social optima of the non-cooperative games will be provided and denoted by "NE" and "Optima", respectively.

We consider a Wyna linear network with 3 BSs (transmitters) and 2 MTs (receivers), as shown in Fig. 3. The 3 BSs are equally spaced with distance $d=20$ meters. The location of MT1 is fixed at the middle of BS1 and BS2. Let $L_{2}$ be the horizontal distance between BS2 and MT2, we let MT2 moves from the right border of BS2 $\left(L_{2}=1\right.$ meter far from BS2) to the middle between BS2 and BS3 $\left(L_{2}=10\right.$ meters far from BS2). The height of each BS is $h_{B S}=3$ meters; the power constraint of each BS is $\bar{P}=1$; the variance of additive white Gaussian noise is set to $\sigma^{2}=0.01$; The propagation loss factor is three. The system performance (spectral efficiency in Bits/Sec/Hz) is averaged over $10^{4}$ realizations of frequency selective fading channels. Fig. 4 shows the average system spectral efficiency (SE) vs. $L_{2}$ (in meters). The NBS outperforms the NE and the Optima of the non-cooperative games. 
BS 1

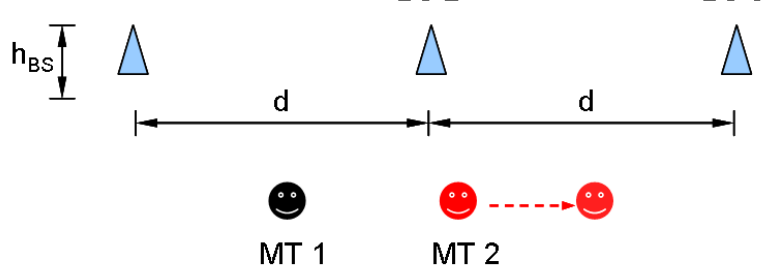

Fig. 3. Illustration of simulation scenario

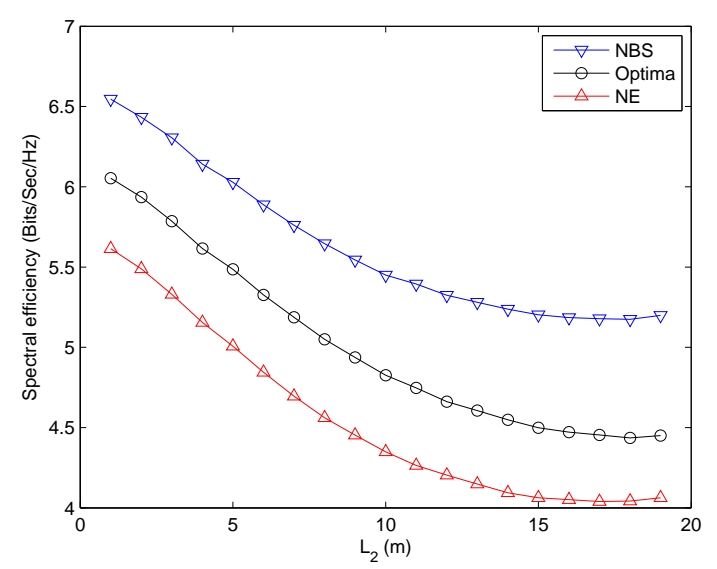

Fig. 4. System spectral efficiency vs. $L_{2}$

Fig. 5 shows the benefit of bargaining (BoB) vs. $L_{2}$. Note that by performing base stations cooperation through Nash bargaining, the overall system performance is improved around $23 \%$ (between $16.5 \%$ and $28 \%$ when $L_{2}$ varies from 1 meter to 10 meters, respectively), comparing to the Nash equilibrium in the non-cooperative game setting. In Fig. 6, we compare the average spectral efficiency for the three BSs when different game solutions are applied. As expected, Nash bargaining provides a more "fair" and efficient solution compared to the non-cooperative game solutions: NE and Optima. By using NBS scheme, BS1 and BS2 maintain the similar performance as in NE and Optima, but BS3 obtains nearly doubled-performance compare to NE and Optima schemes.

\section{CONClusion ANd FUture WORKS}

In this paper we described the scenario of multiple transmitters communicating with multiple receivers through independent parallel sub-channels as a cooperative game. We mainly study the Nash bargaining game by using KKT conditions and pure potential. A recursive stochastic

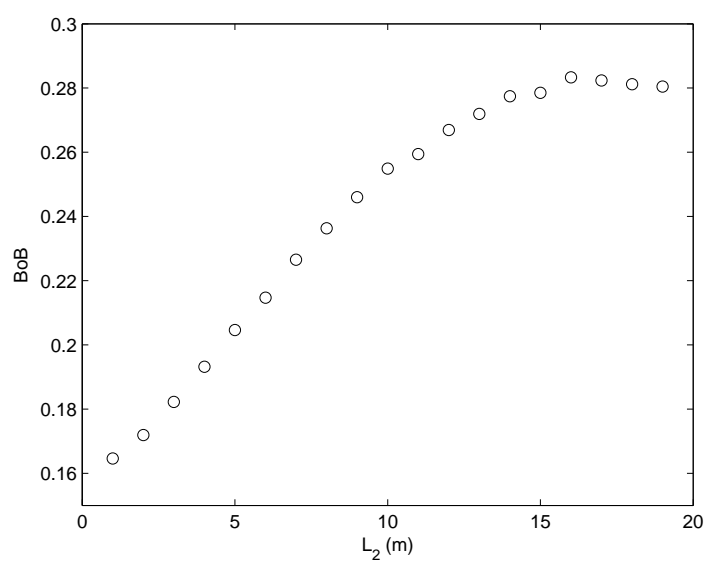

Fig. 5. Benifit of bargaining (BoB) vs. $L_{2}$

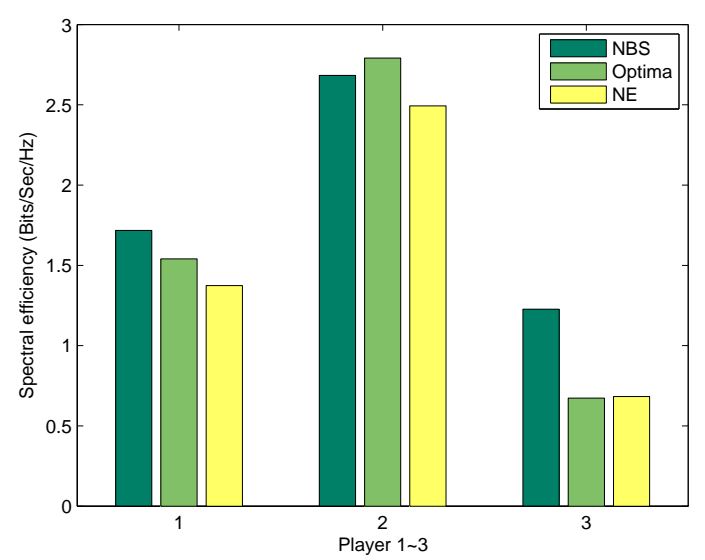

Fig. 6. Fairness for NBS, Optima and NE.

algorithm is proposed to converge to one point as a NBS on the Pareto boundary. Finally, in our simulation results, we show the NBS not only provides a fair power allocation for players, but also outperform the system spectral efficiency compare to NE and Optima solutions (without considering fairness) of the non-cooperative games. Future works will focus on coalition games and dynamic Nash bargaining.

\section{APPENDIX}

\section{A. Proof of Lemma III.1}

Proof: By definition of the disagreement outcome and the feasible payoffs set $\mathcal{F}$, suppose that there exists a feasible rate profile $R$ that component-wise dominates $R^{\min }$, then we must have $R_{m}>R_{m}^{\min }, \forall m$, which implies the existence of $\epsilon_{m}>0$ such that $R_{m}-R_{m}^{\min } \geq \epsilon_{m}$ for all $m$. Let $\bar{\epsilon}$ be the minimum of the $\left\{\epsilon_{m}\right\}_{m \in \mathcal{M}}$. Then, $\bar{\epsilon}>0$ is strictly positive. Let $\epsilon$ such that $0<\epsilon<\bar{\epsilon}$. The supremum over $\mathcal{F}$ must be in the region $R_{m}>R_{m}^{\min }+\epsilon$ for all $m$. Thus, $\forall m, \phi_{\epsilon}\left(R_{m}-R_{m}^{\min }\right)=\log \left(R_{m}-R_{m}^{\min }\right)$ and the constrained maximization of $\sum_{m=1}^{M} \omega_{m} \phi_{\epsilon}\left(R_{m}-R_{m}^{\min }\right)$ coincides with $\sum_{m=1}^{M} \omega_{m} \log \left(R_{m}-R_{m}^{\min }\right)$. Now consider the case where 
the rate profile satisfies the following: there exists a mobile $m$ for which the total rate over all the channels $\bar{R}_{m}$ is strictly lower than the disagreement rate. $R_{m}^{\min }+\epsilon>\bar{R}_{m}$. Then, we show that this configuration cannot be a Nash bargaining solution.

$$
\phi_{\epsilon}\left(\bar{R}_{m}^{\prime}-R_{m}^{\min }\right)=\log (\epsilon)+\frac{\bar{R}_{m}^{\prime}-R_{m}^{\min }-\epsilon}{\epsilon}
$$

and the term $\frac{\bar{R}_{m}^{\prime}-R_{m}^{\min }-\epsilon}{\epsilon}$ is negative. Thus,

$$
\begin{aligned}
c c & :=\sum_{m^{\prime}} \omega_{m^{\prime}} \phi_{\epsilon}\left(\bar{R}_{m^{\prime}}^{\prime}-R_{m^{\prime}}^{\min }\right) \\
& \leq \omega_{m} \log (\epsilon)+\sum_{m^{\prime} \neq m} \omega_{m^{\prime}} \log \left(\bar{R}_{m^{\prime}}-R_{m^{\prime}}^{\min }\right) \\
& <\sum_{m^{\prime}} \omega_{m^{\prime}} \log \left(R_{m^{\prime}}-R_{m^{\prime}}^{\min }\right)
\end{aligned}
$$

Therefore, $\bar{R}$ is never the maximizer of (6). Thus, for $\bar{R}$ to be a maximizer, it must satisfy $\bar{R}_{m}^{\prime}>R_{m, \min }$ for all $m$ i.e $\forall m, \phi_{\epsilon}\left(R_{m}-R_{m}^{\min }\right)=\log \left(R_{m}-R_{m}^{\min }\right)$ and the constrained maximization of $\sum_{m=1}^{M} \omega_{m} \phi_{\epsilon}\left(R_{m}-R_{m}^{\min }\right)$ over $\mathcal{F}$ coincides with $\sum_{m=1}^{M} \omega_{m} \log \left(R_{m}-R_{m}^{\min }\right)$ over $\mathcal{F} \cap\left\{R, \bar{R}_{m}>R_{m}^{\min }\right\}$. This completes the proof.

\section{B. Proof of Proposition III.1}

Proof: We will use the proof by contradiction. First, let us assume $\lambda_{n}^{\mathcal{M}}=0$ is true, and we have the corresponding sum-rate constraint

$$
\sum_{m \in \mathcal{M}} R_{m, n}^{\star} \leq \bar{R}_{n}^{\mathcal{M}}
$$

On the one hand, since $\lambda_{n}^{\mathcal{M}}=0$, there must exist at least one player set $\mathcal{J}(\mathcal{J} \neq \mathcal{M})$ satisfying $\lambda_{n}^{\mathcal{J}}>0$, due to the fact $\beta>0$. This implies

$$
\sum_{m \in \mathcal{J}} R_{m, n}^{\star}=\bar{R}_{n}^{\mathcal{J}}
$$

On the other hand, for the complementary player set of $\mathcal{J}$, i.e. $\mathcal{J}^{c}:=\mathcal{M}-\mathcal{J}$, the following constraint must be satisfied

$$
\sum_{m \in \mathcal{J}^{c}} R_{m, n}^{\star} \leq \bar{R}_{n}^{\mathcal{J}^{c}}
$$

From (26) and (27), we obtain

$$
\sum_{m \in \mathcal{M}} R_{m, n}^{\star} \leq \bar{R}_{n}^{\mathcal{J}}+\bar{R}_{n}^{\mathcal{J}^{c}}
$$

which is in contradication with (25), because of the subadditive property, i.e. $\bar{R}_{n}^{\mathcal{M}}=\bar{R}_{n}^{\mathcal{J} \cup \mathcal{J}^{c}}<\bar{R}_{n}^{\mathcal{J}}+\bar{R}_{n}^{\mathcal{J}^{c}}$ (This proof is provided in subsection III-D). Therefore, $\lambda_{n}^{\mathcal{M}}=0$ can not be true. We must have $\lambda_{n}^{\mathcal{M}}>0$ and (11) due to (10).

\section{Proof of Proposition III.2}

Proof: A sum-rate bargaining solution (Nash Bargaining) $R^{*}$ is a Pareto optimal solution for the sumrate maximization. Hence, $R$ satisfies $\sum_{m} R_{m}^{*}=v(\mathcal{M})$. Consider now that a $\mathcal{J}$ of players deviate from $R^{*}$ and form a new coalition with rates $R_{\mathcal{J}}^{\prime}$. The new rate profile is then $\left(R_{\mathcal{J}^{\prime}}^{\prime}, R_{\mathcal{J}^{c}}\right)$. If $R_{\mathcal{J}}^{\prime}$ does not meet the capacity constraints, the payoff of each player will be zero. In the capacity region the total sum rate is constrained to the following inequality:

$$
\sum_{m \in \mathcal{J}} R_{m}^{\prime} \leq v(\mathcal{M})-\sum_{m \in \mathcal{J}^{c}} R_{m}^{\prime}=\sum_{m \in \mathcal{J}} R_{m}
$$

So, the optimal deviation will have the equality

$$
\sum_{m \in \mathcal{J}} R_{m}^{\prime}=\sum_{m \in \mathcal{J}} R_{m}
$$

This means that if a sum-rate of one of the players in $\mathcal{J}$ increases, then there exists another player in the coalition $\mathcal{J}$ for which the sum-rate decreases after deviating. Thus, the coalition is non-profitable, and this holds for any coalition $\mathcal{J} \subseteq \mathcal{M}$. We conclude that the bargaining solutions are strong equilibria of the game $G$.

\section{Proof of Proposition III.3}

Proof: First, remark that if the sum-rate profile $R$ is not in boundary surface of the capacity region, then $\alpha$ is not resilient by deviation by a single player. Hence, $R$ cannot be a coalition-proof equilibrium [22]. This says that a necessary condition for a rate profile to be a strong equilibrium for $G$ is to be in the subset the maximal face of the sum-rate capacity region. It is easy to see that this last condition is also sufficient: if $k$ players deviate simultaneously from the rate profile $R$ and form a coalition then, the sum-rate constraints of the new coalition is bounded $\sum_{m \in \mathcal{J}} R_{m}^{\prime} \leq v(\mathcal{M})-\sum_{m \notin \mathcal{J}} R_{m}$. The players in the coalition with a lower rate $R_{m}^{\prime} \leq R_{m}$ do not benefit to be member of the coalition (Shapley criterion of membership of coalition does not hold), and this holds for any $J \subseteq \mathcal{M}$ with cardinality of $\mathcal{J} \geq 1$. This completes the proof.

\section{REFERENCES}

[1] M. Debbah, "Mobile Flexible Networks: The challenges ahead", in Proc. International Conference on Advanced Technologies for Communications, Viet Nam (2008), pp. 3-7, Oct. 2008.

[2] J. Bingham, "Multicarrier Modulation for Data Transmission: An Idea Whose Time Has Come", IEEE Communications Magazine, vol. 28, no. 5, pp. 514, May 1990.

[3] B. Muquet, "Novel Receiver and Decoding Schemes for Wireless OFDM Systems with Cyclic Prefix or Zero-padding", PhD dissertation, École Nationale Supérieure des Télécommunications, June 2001.

[4] B. Muquet, Z. Wang, G. B. Giannakis, M. de Courville, P. Duhamel, "Cyclic-Prefixing or Zero-Padding for Wireless Multicarrier Transmissions?" IEEE Trans. on Communications, vol. 50, pp. 2136-2148, Dec. 2002.

[5] C. E. Shannon, "A mathematical theory of communication", Bell System Technical Journal, vol. 27, pp. 379-423 and 623-656, July and October, 1948.

[6] S. Boyd and L. Vandenberghe, Convex Optimization, Cambridge University Press, 2004.

[7] T. M. Cover and J. A. Thomas, Elements of Information Theory, Wiley, 1991.

[8] A.J. Goldsmith and P.P. Varaiya, "Capacity of Fading Channels with Channel Side Information”, IEEE Trans. on Information Theory, vol. 43, pp. 1986-1992, 1997.

[9] D. Tse and P. Viswanath, Fundamentals of Wireless Communication, Cambridge University Press, 2005.

[10] R. Horst and H. Tuy, Global optimization: deterministic approaches, 2nd ed. Berlin; New York: Springer-Verlag, 1993. 
[11] R. Cendrillon, W. Yu, M. Moonen, J. Verlinden and T. Bostoen, "Optimal Multi-user Spectrum Management for Digital Subscriber Lines", IEEE International Conference on Communication (ICC), vol. 1, pp. 1-5, 2004.

[12] D. Gesbert and M. S. Alouini, "How Much Feedback is MultiUser Diversity Really Worth?", IEEE International Conference on Communications (ICC), Paris, France, vol. 1, pp. 234-238, June 2004.

[13] D. Fudenberg and J. Tirole, Game Theory, MIT Press, 1991.

[14] W. Yu, G. Ginins and J. M. Cioffi, "Distributed Multiuser Power Control for Digital Subscriber Lines", IEEE Journal on Selected Areas in Communications, vol. 20, no. 5, June 2002.

[15] Harold J. Kushner G. George Yin, Stochastic Approximation and Recursive Algorithms and Applications, 2003

[16] Z. Q. Luo and J. S. Pang, "Analysis of Iterative Waterfilling Algorithm for Multiuser Power Control in Digital Subscriber Lines", EURASIP Journal on Applied Signal Processing, vol. 2006, Article ID 24012, 10 pages, 2006.

[17] G. He, S. Betz and M. Debbah, "Deployment Design of Selfish OFDM Hot-spots", submitted to ISIT, Jan. 2009.

[18] Z. Han, Z. Ji and K. J. R. Liu, "Fair Multiuser Channel Allocation for OFDMA Networks Using Nash Bargaining Solutions and Coalitions", IEEE Transactions on Communications, vol. 53, no. 8, Aug. 2005.

[19] K. W. Shum and C. W. Sung, "On the Fairness of Rate Allocation in Gaussian Multiple Access Channel and Broadcast Channel", http://arxiv.org/abs/cs/0611015v1, submitted on Nov. 2006.

[20] A. Leshem and E. Zehavi, "Cooperative game theory and the Gaussian interference channel", http://arxiv.org/abs/0708.0846, submitted on 6 Aug. 2007.

[21] R. J. Aumann, "Acceptable Points in General Cooperative n-Person Games", in Contributions to the Theory of Games IV, Annals of Mathematics Study 40, edited by A. W. Tucker and R. D. Luce, Princeton: at the University Press, pp. 287-324, 1959.

[22] D. Moreno and J. Wooders, "Coalition-Proof Equilibrium", Games and Economic Behavior, vol. 17, pp. 80-112, 1996. 